Article

\title{
Multivariable Static Output Feedback Control of a Binary Distillation Column using Linear Matrix Inequalities and Genetic Algorithm
}

\author{
Kalpana R. ${ }^{1}$, Harikumar K. ${ }^{2 *}$, Senthilkumar J. ${ }^{1}$, Balasubramanian G. ${ }^{1}$, and Abhay S. Gour ${ }^{3}$ \\ 1 School of Electrical and Electronics Engineering, SASTRA University, Thanjavur, Tamilnadu, India; e-mail: \\ kalpana.elango@src.sastra.edu; jsenthilkumar18@src.sastra.edu; balu_eie@eie.sastra.edu. \\ 2 School of Electrical and Electronic Engineering, Nanyang Technological University, Singapore; e-mail: \\ harikumar100@gmail.com; kharikumar@ntu.edu.sg. \\ 3 Centre for Cryogenic Engineering, Indian Institute of Technology, Kharagpur, India; e-mail: \\ abhay.s.gour@gmail.com \\ * Correspondence: harikumar100@gmail.com; kharikumar@ntu.edu.sg; Tel.: +6590547402
}

\begin{abstract}
The current work addresses the control of two-input two-output (TITO) Wood and Berry model of a binary distillation column. The controller design problem is formulated in terms of multivariable $H_{\infty}$ control synthesis. The controller structure takes the form of simplest static output feedback (SOF) control. The controller synthesis is performed using a hybrid approach of blending linear matrix inequalities (LMI) and genetic algorithm (GA). The performance of the static output feedback controller is compared with three other controllers designed for Wood and Berry model available in the literature. The first simulation study is performed for the case of tracking a unit step command in the presence of a step change in output disturbance. A second simulation study is performed for rejecting a change in sinusoidal output disturbance.
\end{abstract}

Keywords: Distillation column, Disturbance rejection, Genetic algorithm, $H_{\infty}$ control, Linear matrix inequalities, Static output feedback.

\section{Introduction}

Multivariable chemical processes are arduous to control due to the coupling between multiple input channels and multiple output channels. Distillation control is a challenging problem due to inherent non-linear nature of the process, severe coupling between various inputs and outputs, nonstationary behavior of the process and effect of unmeasured disturbances. In this paper, we address the issue of multivariable nature of the distillation control having interaction among different control loops and the effect of unmeasured output disturbances. Wood and Berry proposed a popular two-input two-output (TITO) transfer function model of a pilot scale binary distillation column [1]. A plethora of multivariable control techniques are available in the literature, applied to TITO model of binary distillation column proposed by Wood and Berry, [2]-[9]. A method based on the static decoupler design using the steady state gain matrix is proposed in [2]. The decentralized proportional-integral-derivative (PID) controller designed for the decoupled process is then combined with the static decoupler to yield a multivariable controller. A similar methodology of decoupling followed by design of decentralized controllers are discussed in [3] - [9]. The performance of the decentralized controllers depends upon the degree of decoupling achieved. Under the ubiety of strong coupling between the different control loops, the performance of decentralized controller degrades. Multivariable control design techniques inherently handle the coupling related issues found common 
in distillation control.

In this paper, a multivariable static output feedback (SOF) controller is designed using a hybrid approach of blending linear matrix inequalities (LMI) and genetic algorithm (GA). Static output feedback control has found a lot of applications due to the ease in practical implementation [10] - [14]. LMI is a powerful tool for synthesizing output feedback controllers satisfying multiple requirements. Multiple objectives like robust pole placement, $H_{2} / H_{\infty}$ performance measures and frequency domain specifications can be easily realized through a system of LMI's [15] - [20]. The conventional solution of LMI's utilizes convex programming methods [21]. Multi-objective design requirements often leads to a system of bilinear matrix inequalities (BMI). In this paper, BMI is transformed to LMI by restructuring the additional slack variable of BMI as an input variable to GA. The solution provided by GA evolves over several iterations based on probabilistic rules and leads to an optimal/suboptimal solution [22]. The paper is organized as follows. Section 2, discuss about the transfer function and state space model of Wood and Berry distillation column. A multivariable $H_{\infty}$ control formulation is given in section 3 . An algorithm for the synthesis of SOF controller is given in section 4 . Section 5 discusses the simulation results, comparing the performance of SOF controller with other three controllers available in the literature for Wood and Berry distillation column. Section 6 concludes the paper.

\section{Model of Binary Distillation Process}

The TITO transfer function model for methanol-water separation in a distillation column is given in equation 1, [1]. The controlled variables (outputs) are the composition of top product $\left(Y_{1}\right)$ and bottom product $\left(Y_{2}\right)$, expressed in percentage of methanol in weight. The manipulated variables (control inputs) are the reflux steam flow rate $\left(U_{1}\right)$ and the reboiler steam flow rate $\left(U_{2}\right)$ in the units of $1 \mathrm{~b} / \mathrm{min}$. All the time constants are expressed in the unit of minutes (min).

$$
\left(\begin{array}{l}
Y_{1}(s) \\
Y_{2}(s)
\end{array}\right)=\left(\begin{array}{cc}
\frac{12.8 e^{-s}}{16.7 s+1} & \frac{-18.9 e^{-3 s}}{21 s+1} \\
\frac{6.6 e^{-7 s}}{10.9 s+1} & \frac{-19.4 e^{-3 s}}{14.4 s+1}
\end{array}\right)\left(\begin{array}{l}
U_{1}(s) \\
U_{2}(s)
\end{array}\right)
$$

Using a first order Pade-approximation for time delay in equation 1 yields a rational transfer function for the distillation column model as given in equation 2.

$$
\left(\begin{array}{l}
Y_{1}(s) \\
Y_{2}(s)
\end{array}\right)=\left(\begin{array}{ll}
g_{11}(s) & g_{12}(s) \\
g_{21}(s) & g_{22}(s)
\end{array}\right)\left(\begin{array}{l}
U_{1}(s) \\
U_{2}(s)
\end{array}\right)
$$

The expression for the terms $g_{11}(s)$ to $g_{22}(s)$ are given in equations from 3 to 6 .

$$
\begin{aligned}
& g_{11}(s)=\frac{12.8(-0.5 s+1)}{(16.7 s+1)(0.5 s+1)} \\
& g_{12}(s)=\frac{-18.9(-1.5 s+1)}{(21 s+1)(1.5 s+1)} \\
& g_{21}(s)=\frac{6.6(-3.5 s+1)}{(10.9 s+1)(3.5 s+1)} \\
& g_{22}(s)=\frac{-19.4(-1.5 s+1)}{(14.4 s+1)(1.5 s+1)}
\end{aligned}
$$

The state space model of the system given in equation 2 is given in equations 7 and 8 . The $A$ matrix for the state space model is given in equation 9 . The $B$ matrix for the state space model is given in equation 10 and $C$ matrix is given in equations 11 .

$$
\dot{X}=A X+B U
$$




$$
Y=C X
$$

$$
\begin{aligned}
& A=\left(\begin{array}{cccccccc}
-2.06 & -0.479 & 0 & 0 & 0 & 0 & 0 & 0 \\
0 & 0 & 0 & 0 & 0 & 0 & 0 & 0 \\
0.25 & 0 & 0 & 0 & 0 & 0 & 0 & 0 \\
0 & 0 & -0.378 & -0.210 & 0 & 0 & 0 & 0 \\
0 & 0 & 0.125 & 0 & -0.714 & -0.127 & 0 & 0 \\
0 & 0 & 0 & 0 & 0.25 & 0 & 0 & 0 \\
0 & 0 & 0 & 0 & & & & \\
0 & 0 & 0 & 0 & 0 & 0 & -0.736 & -0.185 \\
0 & 0 & 0 & 0 & 0 & 0 & 0.25 & 0
\end{array}\right) \\
& B=\left(\begin{array}{ll}
2 & 0 \\
0 & 0 \\
1 & 0 \\
0 & 0 \\
0 & 2 \\
0 & 0 \\
0 & 2 \\
0 & 0
\end{array}\right) \\
& C=\left(\begin{array}{cccccccc}
-0.383 & 3.066 & 0 & 0 & 0.45 & -1.2 & 0 & 0 \\
0 & 0 & -0.606 & 1.384 & 0 & 0 & 0.674 & -1.796
\end{array}\right)
\end{aligned}
$$

46 3. Multivariable $H_{\infty}$ control formulation

47 Optimization in $H_{\infty}$ space is suitable for systems having uncertainty in dynamics and disturbances. A

48 diagram representing the $H_{\infty}$ control formulation for the binary distillation column is given in figure 1 .

49 Along with the weighted sensitivity function $(S)$, weighted complementary sensitivity function (T) is

so also minimized leading to multi-objective design problem [24]. Generic form of the weights $W_{1}(s)$ and

${ }_{51} W_{2}(s)$ are given in the equations 12 and 13 respectively. The weighting function $W_{2}(s)$ is taken as high

52 pass filter to attenuate the detrimental effects of unmodeled dynamics at higher frequencies. To retain

53 good tracking and disturbance rejection properties at low frequencies, an ideal choice for the weighting

54 function $W_{1}(s)$ is a low pass filter. The performance outputs to be minimized are $Z=\left[Z_{1}, Z_{2}\right]$ and are

55 given in equations 14 and 15 respectively. 


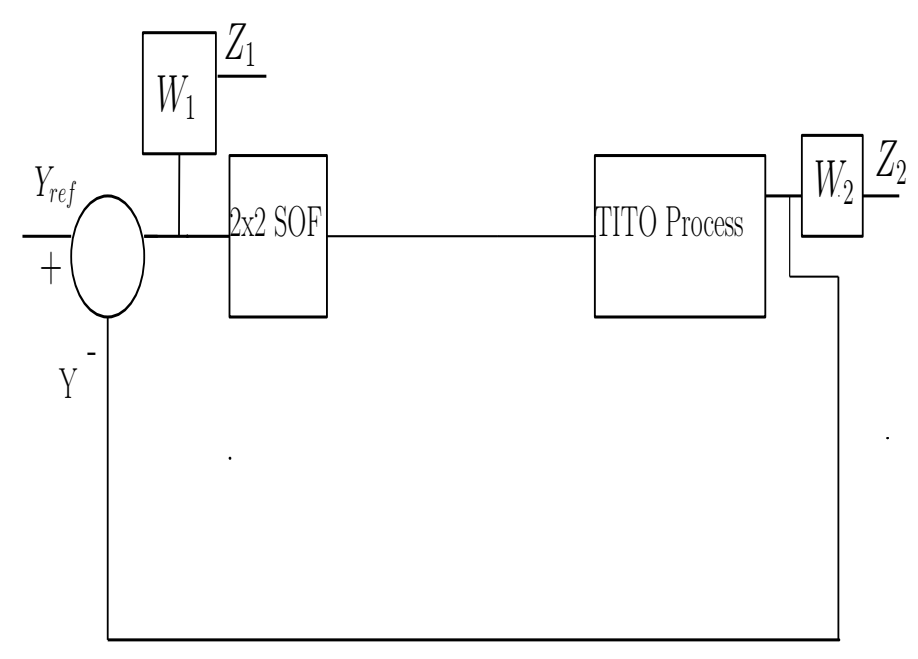

Figure 1. Diagram representing $H_{\infty}$ control formulation.

$$
\begin{gathered}
W_{1}(s)=\left(\begin{array}{cc}
W_{e_{1}}(s) & 0 \\
0 & W_{e_{2}}(s)
\end{array}\right) \\
W_{2}(s)=\left(\begin{array}{cc}
W_{y_{1}}(s) & 0 \\
0 & W_{y_{2}}(s)
\end{array}\right) \\
Z_{1}=\left[Z_{e_{1}}, Z_{e_{2}}\right]^{T} \\
Z_{2}=\left[Z_{y_{1}}, Z_{y_{2}}\right]^{T}
\end{gathered}
$$

56 The linear state space equation for the generalized plant are given in equations 16 to 19. Generalized 57 plant is obtained by augmenting the state space model of binary distillation column given in equations

${ }_{58} 7$ to 11 with the weighting transfer functions $W_{1}(s)$ and $W_{2}(s)$. In equations 16 to $19, X_{t g}$ is the state

${ }_{59}$ vector of the generalized plant, $Y$ is the vector of measurements $\left(Y_{1}\right.$ and $\left.Y_{2}\right), U_{t c}$ is the vector of control

6o inputs $\left(U_{1}\right.$ and $\left.U_{2}\right)$ and $W_{t d}$ is the vector of disturbance inputs. The disturbance inputs $W_{t d}$ corresponds

${ }_{61}$ to the reference inputs $Y_{1 \text { ref }}$ and $Y_{2 \text { ref }}$.

$$
\dot{X}_{t g}=A_{t g} X_{t g}+B_{t u} U_{t c}+B_{t w} W_{t d}
$$

62

$$
Z_{1}=C_{t 1} X_{t g}+D_{t 11} U_{t c}+D_{t 12} W_{t d}
$$

$$
Z_{2}=C_{t 2} X_{t g}+D_{t 21} U_{t c}+D_{t 22} W_{t d}
$$

$$
Y=C_{t} X_{t g}
$$


67

Selection of weighting transfer functions $W_{1}(s)$ and $W_{2}(s)$ follow the guidelines presented in [23]. The low pass filter weights $W_{e_{1}}(s)$ and $W_{e_{2}}(s)$ are given in equations 20 and 21 respectively. The high pass filter weights $W_{y_{1}}(s)$ and $W_{y_{2}}(s)$ are given in equations 22 and 23 respectively.

$$
\begin{gathered}
W_{e_{1}}(s)=\frac{0.1}{(s+0.2)} \\
W_{e_{2}}(s)=\frac{0.1}{(s+0.3)} \\
W_{y_{1}}(s)=\frac{0.01(s+0.1)}{(s+2)} \\
W_{y_{2}}(s)=\frac{0.01(s+0.1)}{(s+3)}
\end{gathered}
$$

The objective of the control design problem is to synthesis a SOF controller with the structure, $U_{t c}=K Y$ that minimizes the norm given in equation 24.

$$
\left\|T_{z w_{t d}}\right\|_{\infty}=\left\|\left(\begin{array}{c}
W_{1} S \\
W_{2} T
\end{array}\right)\right\|_{\infty}
$$

71

72

\section{Static output feedback controller synthesis using LMI}

Applying SOF control $U_{t c}=K Y$ for the system given in equations 16 to 19 , the resulting state space model for the closed loop generalized plant are given in equations 25 to 27.

$$
\dot{X}_{t g}=\left(A_{t g}+B_{t u} K C_{t}\right) X_{t g}+B_{t w} W_{t d}
$$

$$
Z_{1}=\left(C_{t 1}+D_{t 11} K C_{t}\right) X_{t g}+D_{t 12} W_{t d}
$$

$$
Z_{2}=\left(C_{t 2}+D_{t 21} K C_{t}\right) X_{t g}+D_{t 22} W_{t d}
$$

Let $A_{c l t}=A_{t g}+B_{t u} K C_{t}$ be the closed loop system matrix. The Lyapunov condition for stability of the closed loop system can be written as the following matrix inequality given in equation 28, [17]. In equation 28, $Q=A_{c l t}{ }^{T} P+P A_{c l t}, M=N B_{t u} B_{t u}{ }^{T} P$ and $R=B_{t u}^{T} P+K C_{t}, P$ is a positive definite matrix and $N$ is any square matrix. The matrix inequality given in equation 28 is bilinear. The bilinear matrix inequality (BMI) in equation 28 can be converted into LMI by fixing one of the unknowns $P$ or $N$. Since there is a constraint on $P(P>0), N$ is taken as a decision variable for genetic algorithm (GA). Choosing $N$ as the decision variable for GA instead of $P$ is less conservative and results in a enlarged search space. Multiple choices for $N$ is selected by GA and the resultant LMI is solved by minimizing a performance index. The performance index is given in equation 29 .

$$
\begin{gathered}
\left(\begin{array}{cc}
Q-M-M^{T}+N B_{t u} B_{t u}{ }^{T} N & R^{T} \\
R & -I
\end{array}\right)<0 \\
J_{P I}=q_{t 1}(100)+q_{t 2}\left(\left\|T_{z w_{t d}}\right\|_{\infty}\right)
\end{gathered}
$$


82

The variables $q_{t 1}$ and $q_{t 2}$ in equation 29 , takes the binary value 0 or 1 based on closed loop stability requirements. The binary value of variables $q_{t 1}$ and $q_{t 2}$ are obtained as follows. Let $\gamma_{c l i}$ denote the $i^{\text {th }}$ eigenvalue of the closed loop system matrix $A_{c l t}$, let $n_{\text {gclt }}$ be the dimension of $A_{c l t}$ and $\operatorname{Re}(\gamma)$ denotes the real part of the complex variable $\gamma$.

CASE I IF $\operatorname{Re}\left(\gamma_{c l i}\right)>=0$, for any $\mathrm{i}=1,2,3, \ldots . ., n_{\text {gclt }}$, THEN $q_{t 1}=1$ and $q_{t 2}=0$.

CASE II IF $\operatorname{Re}\left(\gamma_{c l i}\right)<0, \forall \mathrm{i}=1,2,3, \ldots . ., n_{g c l t}$, THEN $q_{t 1}=0$ and $q_{t 2}=1$.

Therefore, either $J_{P I}=100$ or $0<J_{P I}<1$ (since $\left\|T_{z w_{t d}}\right\|_{\infty} \in(0,1)$ ). The SOF control gains are found iteratively using the algorithm given below.

1. Choose $N$ as decision variable and $J_{P I}$ as the performance index for $G A$.

2. Solve the LMI given in equation 28 to obtain $P$ and $K$ for the value of $N$ given by $G A$.

3. Evaluate the performance index $J_{P I}$, given in equation 29.

4. Iterate the steps 1,2 and 3 until $J_{P I}$ is minimized.

\section{Numerical simulation results and discussion}

For solving the LMI given by the equation 28 , the matrix $N$ is set to a diagonal matrix. Table 1 gives the initialization details of the genetic algorithm toolbox in MATLAB 7.5. The SOF gain obtained is given in equation 30 with $\left\|T_{z w_{t d}}\right\|_{\infty}=0.498$. A comparison of open loop poles and closed loop poles of the system given in equations 7 to 11 is given in Table 2. Please note that there is no one to one correspondence between the open loop and closed loop poles given in Table 2. The SOF gain stabilizes the closed loop system and also minimizes $\left\|T_{z w_{t d}}\right\|_{\infty}$.

Table 1. Initialization of GA toolbox in MATLAB 7.5.

\begin{tabular}{cc}
\hline Attibute & Value \\
\hline Population size & 500 \\
Population range & {$[-20,20]$} \\
Number of generations & 100 \\
Selection function & Stochastic uniform \\
Crossover function & Scattered \\
Mutation function & Gaussian \\
\hline
\end{tabular}

$$
K=\left(\begin{array}{ll}
-0.8145 & 0.6059 \\
-0.2200 & 0.3025
\end{array}\right)
$$

Table 2. Open loop and closed loop poles

\begin{tabular}{cc}
\hline Open loop poles & Closed loop poles \\
\hline-0.0599 & $-0.9807-1.1092 \mathrm{i}$ \\
-0.2857 & $-0.2763+0.2829 \mathrm{i}$ \\
-0.0917 & $-0.2763-0.2829 \mathrm{i}$ \\
-0.6667 & -0.6667 \\
-0.6667 & -0.0405 \\
-0.0694 & $-0.0998+0.0395 \mathrm{i}$ \\
-0.0476 & $-0.0998-0.0395 \mathrm{i}$ \\
\hline
\end{tabular}

A comparative study is conducted to evaluate the performance of the SOF controller with the other controllers designed for TITO Wood and Berry distillation column given in [2] to [4]. The model 
given in equation 1 is simulated in SIMULINK for a time duration of 100 minutes. As a first case, a step change in output disturbance is given in both $Y_{1}$ and $Y_{2}$ from a time instant of 50 minutes. The objective is to track a unit step command in both $Y_{1}$ and $Y_{2}$ channels simultaneously in the presence of a step output disturbance acting in both of the output channels. The performance is quantified in terms of integral square error (ISE) in tracking $Y_{1}$ and $Y_{2}$ and also the integral square value (ISV)of control inputs $U_{1}$ and $U_{2}$. An expression for ISE and ISV are given in the equations 31 and 32 respectively (similar equations are used for $Y_{2}$ and $U_{2}$ ). Please note that the integration is performed numerically using SIMULINK with a fixed time step used for simulation.

$$
\begin{gathered}
I S E=\int_{0}^{100}\left(Y_{1}-\Upsilon_{1 r e f}\right)^{2} d t \\
I S V=\int_{0}^{100} U_{1}^{2} d t
\end{gathered}
$$

Figure 2 gives the output $Y_{1}$ and figure 3 gives the output $Y_{2}$ respectively of various controllers in tracking a unit step command in both of the output channels $\left(Y_{1}\right.$ and $\left.Y_{2}\right)$, in the presence of a unit step change in output disturbance in both of the output channels. The solid blue curve shows the response of the SOF controller given in equation 30. The response of the controllers presented in [2], [3] and [4] is given by black curve (dashed), brown curve (dotted) and green curve (dash-dot) respectively. After the introduction of output disturbance at a time instant of 50 minutes, the response of SOF controller is superior in terms of lowest undershoot and smallest settling time when compared to other controllers. The steady state error for tracking is poor for SOF controller for initial 50 minutes due to the lack of integrator. The performance metric ISE and ISV is given in Table 3 for the case of tracking a unit step command in both of the output channels $\left(Y_{1}\right.$ and $\left.Y_{2}\right)$, in the presence of a unit step change in output disturbance in both of the output channels. The performance of the controllers designed in [3] and [4] are similar, but consumes very large control input when compared to SOF controller. Clearly, the SOF controller outperforms the one designed in [2]. Unmeasured output sinusoidal disturbances of very small frequencies are also prominent in process control applications. Figure 4 gives the output $Y_{1}$ and figure 5 gives the output $Y_{2}$ respectively of various controllers, in the presence of a sinusoidal change in output disturbance of frequency $0.1 \mathrm{rad} / \mathrm{min}$ in both of the output channels. The performance metric ISE and ISV is given in Table 4 for the case of rejecting a sinusoidal change in output disturbance of frequency $0.1 \mathrm{rad} / \mathrm{min}$ in both of the output channels. The sinusoidal disturbance rejection property of SOF controller is comparable to that of the controller designed in [3] and [4] and superior to the one designed in [2]. From Table 4, clearly the control effort (ISV of $U_{1}$ ) is on the lower side for SOF controller when compared to other controllers. 


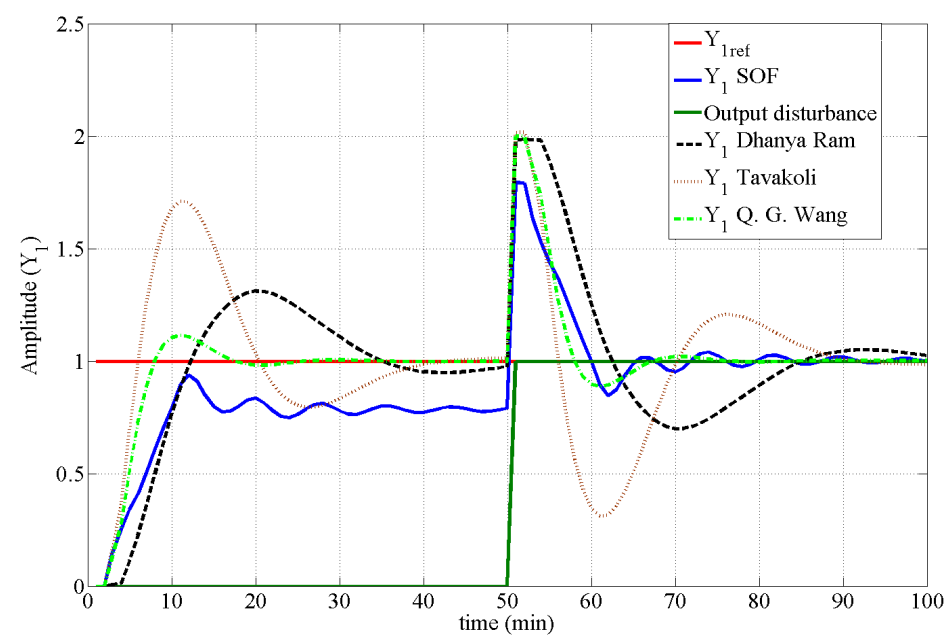

Figure 2. Comparison of the performance of various controllers under step change in output disturbance $\left(Y_{1}\right)$.

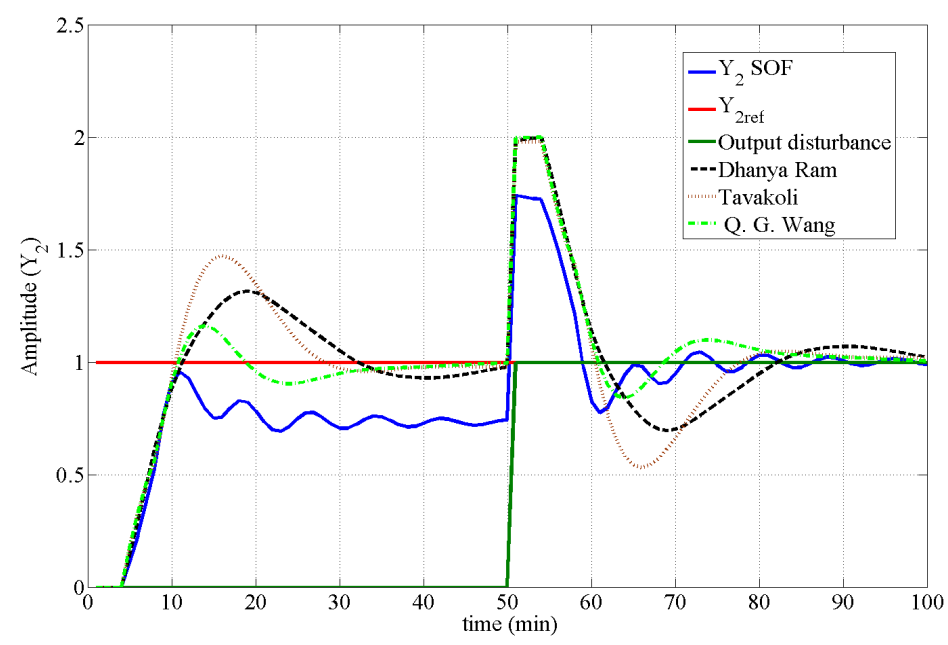

Figure 3. Comparison of the performance of various controllers under step change in output disturbance $\left(Y_{2}\right)$.

Table 3. Results for a step change in output disturbance.

\begin{tabular}{ccccc}
\hline Controller & ISE $\left(Y_{1}\right)$ & ISE $\left(Y_{2}\right)$ & ISV $\left(U_{1}\right)$ & ISV $\left(U_{2}\right)$ \\
\hline SOF Controller & 7.91 & 10.91 & 0.206 & 0.161 \\
Dhanya Ram & 13.28 & 12.31 & 0.100 & 0.283 \\
Tavakoli & 6.20 & 10.61 & 0.348 & 0.183 \\
Wang & 6.23 & 10.64 & 0.344 & 0.187 \\
\hline
\end{tabular}




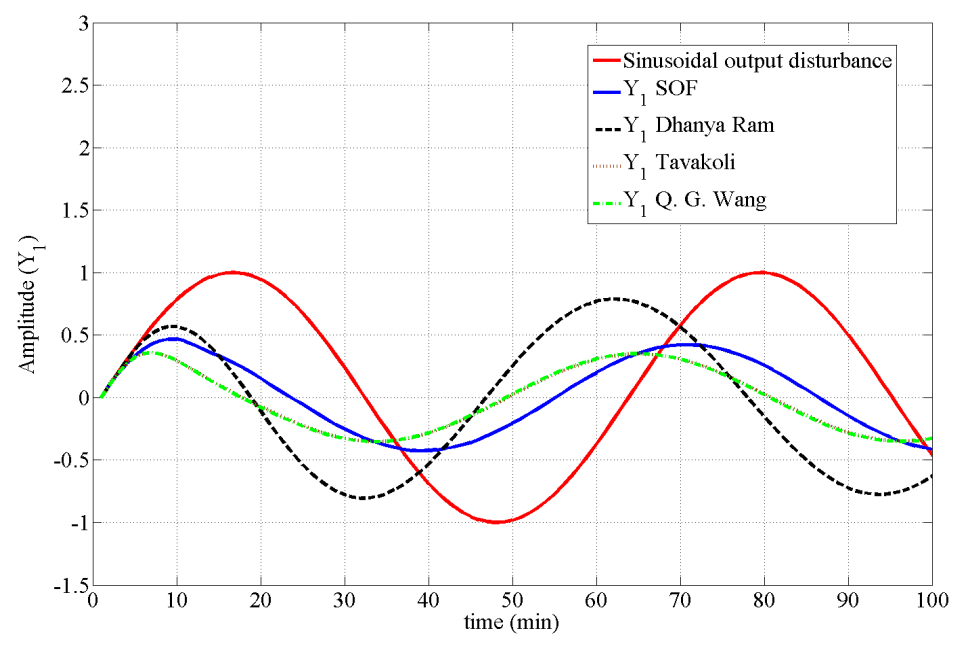

Figure 4. Comparison of the performance of various controllers under sinusoidal change in output disturbance $\left(Y_{1}\right)$.

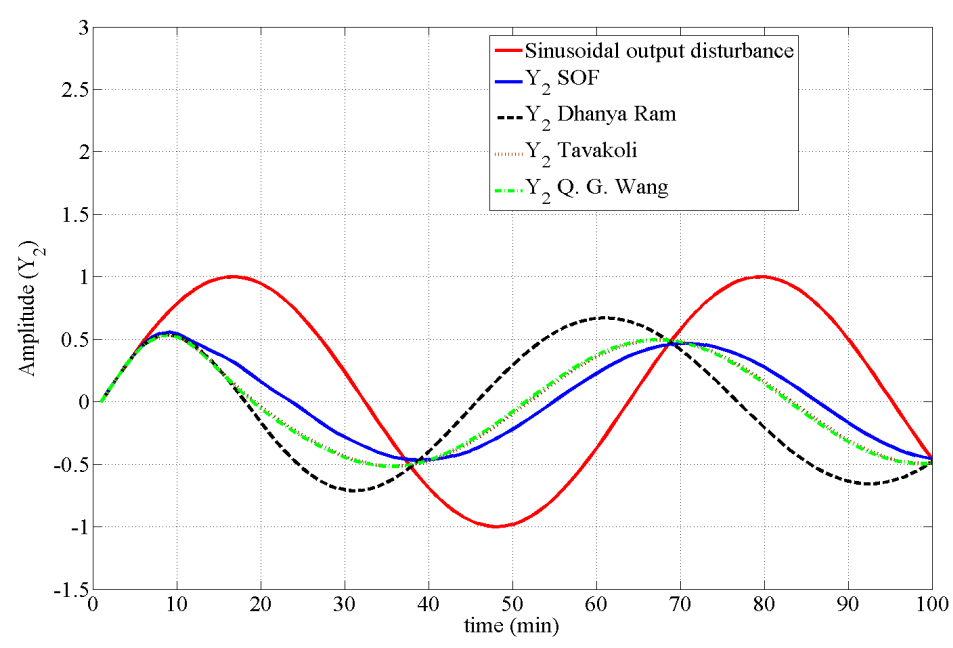

Figure 5. Comparison of the performance of various controllers under sinusoidal change in output disturbance $\left(Y_{2}\right)$.

Table 4. Results for sinusoidal change in output disturbance of frequency $0.10 \mathrm{rad} / \mathrm{min}$.

\begin{tabular}{ccccc}
\hline Controller & ISE $\left(Y_{1}\right)$ & ISE $\left(Y_{2}\right)$ & ISV $\left(U_{1}\right)$ & ISV $\left(U_{2}\right)$ \\
\hline SOF Controller & 9.10 & 11.36 & 0.177 & 0.127 \\
Dhanya Ram & 29.98 & 22.65 & 0.309 & 0.507 \\
Tavakoli & 6.26 & 12.92 & 0.486 & 0.119 \\
Wang & 6.35 & 13.03 & 0.481 & 0.128 \\
\hline
\end{tabular}

\section{Conclusions}

Design of multivarable static output feedback controller for a binary distillation column is presented in this paper. The method adopted for controller synthesis is generic and can be applied to any multivariable systems with unstable poles or non-minimum phase zeros. The designed controller 
has excellent disturbance rejection properties with low control effort. The comparative study with other controllers shows the performance and effectiveness of the controller. The simple structure of the controller yields accurate practical implementation when compared to other higher order controllers. Linear matrix inequalities are powerful tool for designing multivariable static output feedback controllers and further design constraints can be easily incorporated to yield better control solutions.

Author Contributions: Kalpana and Harikumar formulated the problem and written the manuscript. Senthilkumar, Balasubramanian and Abhay. S. Gour did the simulations and also edited the manuscript. All the authors have read and approved the manuscript.

Conflicts of Interest: The authors declare no conflict of interest.

\section{Abbreviations}

The following abbreviations are used in this manuscript:

BMI Bilinear matrix inequality

GA Genetic algorithm

ISE Integral square error

ISV Integral square value

LMI Linear matrix inequality

SOF Static output feedback

TITO Two input two output

\section{References}

1. Wood, R. K.; Berry, M. W. Terminal composition control of a binary distillation column. Chemical Engineering Science. 1973, vol. 28, issue 9, pp. 1707-1717.

2. Dhanya Ram, V.; Chidambaram, M. Simple method of designing centralized PI controllers for multivariable systems based on SSGM. ISA Transactions. 2015, vol. 56, pp. 252-260.

3. Tavakoli, S.; Griffin, I.; Fleming, P.J.; Tuning of decentralised PI (PID) controllers for TITO processes. Control Engineering Practice. 2006, vol. 14, issue 9, pp. 1069-1080.

4. Wang, Q. G.; Huang, B.; Guo, X. Auto-tuning of TITO decoupling controllers from step tests. ISA Transactions. 2000, vol. 39, issue 4, pp. 407-418.

5. Iruthayarajan, M. W.; Baskar, S. Evolutionary algorithms based design of multivariable PID controller. Expert Systems with Applications. 2009, vol. 36, issue 5, pp. 9159-9167.

6. Hajare, V. D.; Patre, B. M. Decentralized PID controller for TITO systems using characteristic ratio assignment with an experimental application. ISA Transactions. 2015, vol. 59, pp. 385-397.

7. Nandong, J.; Zang, Z. Multi-loop design of multi-scale controllers for multivariable processes. Journal of Process Control, 2014, vol. 24, issue 5, pp. 600-612.

8. Chang, W. D. A multi-crossover genetic approach to multivariable PID controllers tuning. Expert Systems with Applications, 2007, vol. 33, issue 3, pp. 620-626.

9. Avila, S.T.; Gutiérrez, A.J.; Hahn, J. Analysis of Multi-Loop Control Structures of Dividing-Wall Distillation Columns Using a Fundamental Model. Processes, 2014, vol. 2, pp. 180-199.

10. Wang, N.; Pei, H.; He, Y.; Zhang, Q. Robust $\mathrm{H}_{2}$ static output feedback tracking controller design of longitudinal dynamics of a miniature helicopter via LMI technique. $24^{\text {th }}$ Chinese Control and Decision Conference. 2012, Taiyuyan, China, pp. 346-350. DOI : 10.1109/CCDC2012.6244051.

11. Harikumar, K.; Arun Joseph, Seetharama Bhat, M; Omkar, S.N. Static output feedback control for an integrated guidance and control of a micro air vehicle. Journal of Unmanned System Technology. 2014, vol.2, No. 1, pp. 17-29.

12. Kong, Y.; Zhau, D.; Yang, B.; Shen, T.; Li, H.; Han, K. Static output feedback control for active suspension using PSO-DE/LMI approach. IEEE international Conference on Mechatronics and Automation. 2012, Chengdu, China, pp. 366-370, DOI : 10.1109/ICMA.2012.6282871. 
13. Komnatska, M.M. Flight control system design via. static output feedback: LMI approach. $2^{\text {nd }} I E E E$ International Conference on Actual Problems of Unmanned Air Vehicles Developments. 2013, Keiv, Ukraine, pp. 184-186, DOI : 10.1109 / APUAVD.2013.6705320.

14. Doye, I.N.; Voos, H.; Darouach, M.; Schneider, J.G.; Knauf, N. Static output feedback stabilization of nonlinear fractional order glucose insulin system. IEEE EMBS International Conference on Biomedical Engineering and Sciences, 2012, Langkawi, Malaysia, pp. 589-594. DOI : 10.1109/IECBES.2012.6498043.

15. Scherer, C.; Gahinet, P.; Chilali, M. Multiobjective output feedback control via. LMI optimization. IEEE Transactions on Automatic Control, 1997, vol. 42, no. 7, pp. 896-911.

16. Chilali, M.; Gahinet, P.; Apkarian, P. Robust pole placement in LMI regions. IEEE Transactions on Automatic Control. 1999, vol. 44, no. 12, pp. 2257-2269.

17. Cao, Y.Y.; Lam, J.; Sun, Y.X. Static output feedback stabilization: an ILMI approach. Automatica. 1998, vol. 34, no. 12, pp. 1641-1645.

18. Fujimori, A. Optimization of static output feedback using substitutive LMI formulation. IEEE Transactions on Automatic Control, 2004, vol. 49, no. 6, pp. 995-999.

19. He, Y.; Wang, Q.G. An improved ILMI method for static output feedback control with application to multivariable PID control. 2006, IEEE Transactions on Automatic Control, vol.51, no. 10, pp. 1678-1683.

20. Lee, D. H.; Joo, Y. H.; Kim, S. K. A Proposition of Iterative LMI Method for Static Output Feedback Control of Continuous-Time LTI Systems. International Journal of Control, Automation and Systems. 2016, vol. 14, no. 4, pp. 1-7.

21. Boyd, S.; Ghaoui, L.E.; Feron, E.; Balakrishnan, V. Linear matrix inequalities in system and control theory. SIAM, Studies in Applied Mathematics. 1994, vol. 15, pp. 12-18.

22. Haupt, R.L.; Haupt, S.E. Practical genetic algorithms. Wiley Interscience. 2004, pp. 27-47, 2004.

23. Jiankun, H.; Bohn, C.; Lu, H.R. Systematic $H_{\infty}$ weighting function selection and its application to the real-time control of a vertical take-off aircraft. Control Engineering Practice. 2000, vol. 8, issue 3, pp. 241-252.

24. Skogestad, S.; Postlethwaite, I. Multivariable feedback control, Analysis and design. John Wiley and Sons. 2001, pp. 309-394. 